Document downloaded from:

http://hdl.handle.net/10251/54355

This paper must be cited as:

Morales Martinez, S.; Naranjo Ornedo, V.; Navea, A.; Alcañiz Raya, ML. (2014). ComputerAided Diagnosis Software for Hypertensive Risk Determination Through Fundus Image Processing. IEEE Journal of Biomedical and Health Informatics. 18(6):1757-1763. doi:10.1109/JBHI.2014.2337960.

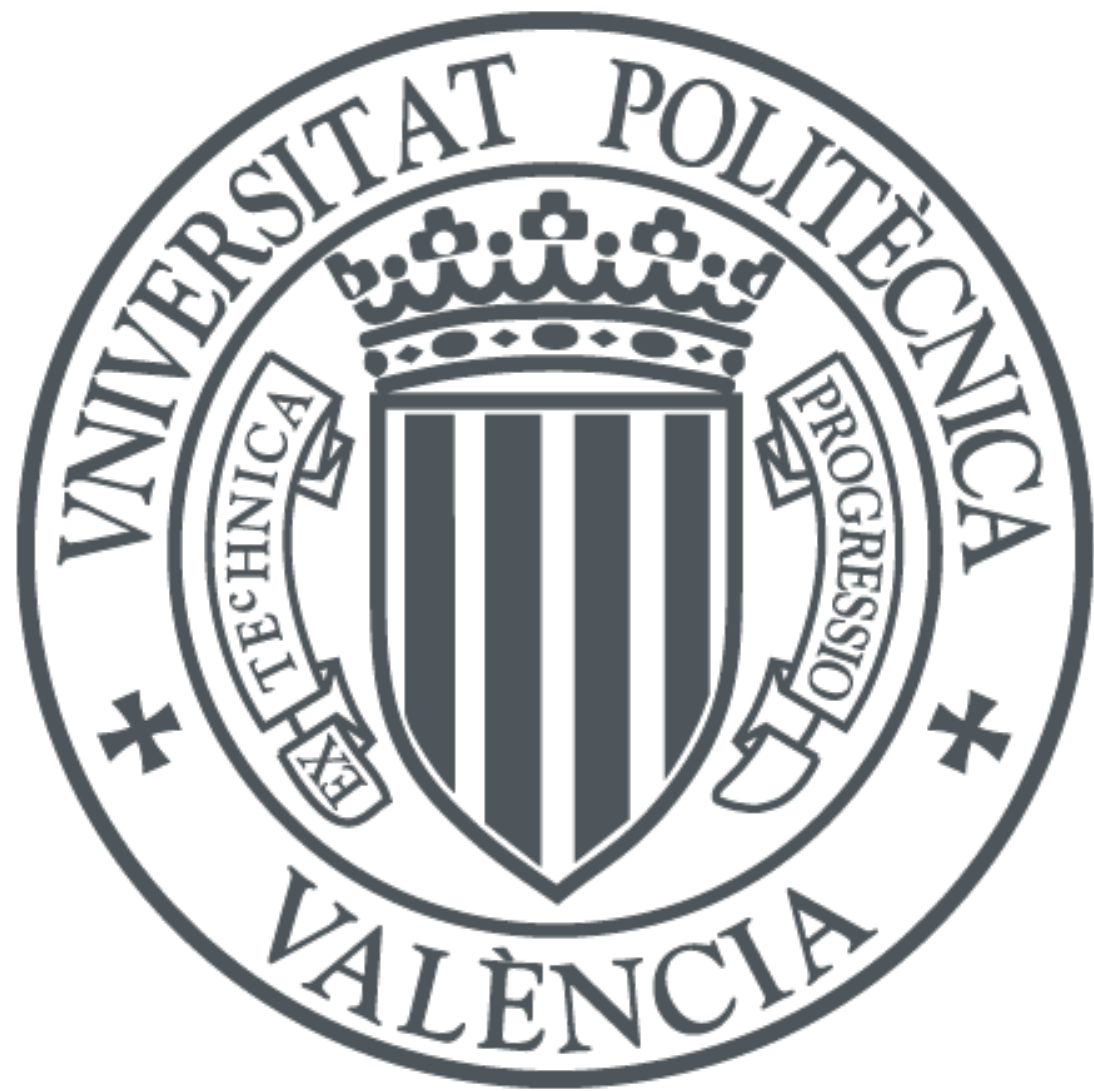

The final publication is available at

http://dx.doi.org/10.1109/JBHI.2014.2337960

Copyright Institute of Electrical and Electronics Engineers (IEEE)

Additional Information

"(c) 20xx IEEE. Personal use of this material is permitted. Permission from IEEE must be obtained for all other users, including reprinting/ republishing this material for advertising or promotional purposes, creating new collective works for resale or redistribution to servers or lists, or reuse of any copyrighted components of this work in other works." 


\title{
Computer-Aided Diagnosis Software for Hypertensive Risk Determination through Fundus Image Processing
}

\author{
Sandra Morales, Valery Naranjo, Amparo Navea, and Mariano Alcañiz
}

\begin{abstract}
The goal of the software proposed in this paper is to assist ophthalmologists in diagnosis and disease prevention, helping them to determine cardiovascular risk or other diseases where the vessels can be altered, as well as to monitor the pathology progression and response to different treatments. The performance of the tool has been evaluated by means of a doubleblind study where its sensitivity, specificity and reproducibility to discriminate between health fundus (without cardiovascular risk) and hypertensive patients has been calculated in contrast to expert ophthalmologist opinion obtained through visual inspection of the fundus image. An improvement of almost $20 \%$ has been achieved comparing the system results with the clinical visual classification.
\end{abstract}

Index Terms-Retinal vessels, retinal vascular tree, vessel calibre, bifurcation angles, hypertension.

\section{INTRODUCTION}

$\mathbf{R}$ ETINAL vasculature is able to indicate the status of other vessels of the human body. Classically, its study is included in the standard screening of any patients with cardiovascular risk and other diseases in which the vessels may be altered inasmuch as it is a non-invasive or minimally invasive procedure.

Nowadays, retinopathies associated with systemic diseases such as diabetes and hypertension are increasingly affecting the population. A direct, regular and complete ophthalmologic examination seems to be the best approach for risk population assessment [1]. However, population growth, ageing, physical inactivity and rising levels of obesity are contributing factors in increasing this type of diseases, so that the number of ophthalmologists required for a direct examination of the risk population is high [2].

Due to high resolution of digital fundus images, they can be automatically processed providing invaluable help to clinicians in diagnosis and disease prevention. Most attempts to automate the process of interpretation of retinal vascular imaging are focused on a specific disease, diabetic retinopathy, a disease of high incidence and a significant risk of blindness that occupies a very important part of the medical-surgical activity

S. Morales, V. Naranjo, and M. Alcañiz are with the Instituto Interuniversitario de Investigación en Bioingeniería y Tecnología Orientada al Ser Humano, Universitat Politècnica de València, I3BH/LabHuman, Camino de Vera s/n, 46022 Valencia, Spain. E-mail: \{smorales, vnaranjo, malcaniz\}@labhuman.com.

M. Alcañiz is also with Ciber, Fisiopatología de Obesidad y Nutrición, CB06/03 Instituto de Salud Carlos III, Spain.

A. Navea is with FISABIO Oftalmología Médica, Bifurcación Pío BarojaGeneral Avilés s/n, 46015 Valencia, Spain. E-mail: navea_amp@gva.es

Manuscript received November 26, 2013. of the ophthalmologic resources [3], [4]. In some of these studies it has been possible to relate the evolution of the disease and the positive or negative response to treatment with retinal vessel calibre [5]-[7]. However, there is not too much experience in the use of these methods to evaluate other types of vascular pathology although fundus photography allows also determination of cardiovascular risk factors [8]. Vascular changes produced in systemic diseases usually induce particular modifications in the vessels, such as changes in the angle of intersection between arteries and veins, and changes in the vessel calibres. Based on these facts, a system capable of detecting the retinal vessels and measuring some geometrical properties from a fundus image has been developed. The goal of the software proposed in this paper is to assist ophthalmologists in diagnosis and disease prevention, helping them to establish objective relations between the different vessels, to determine cardiovascular risk or other diseases where the vessels can be altered, as well as to monitor the pathology progression and response to different treatments. This tool has been applied in a clinical study in order to evaluate sensitivity, specificity and reproducibility of the developed system to discriminate between a normal vascularization and cardiovascular pathology in contrast to the opinion of an expert ophthalmologist obtained through visual inspection of the fundus image.

Regarding vessel extraction techniques present in the literature, they can be mainly grouped into four categories: edge detectors, matched filters, pattern recognition techniques and morphological approaches. A more extensive classification can be found in [9]. Most edge detection algorithms assess changes between pixels values by calculating image gradient magnitude and then it is thresholded to create a binary edge image [10], [11]. Matched filters are filters rotated in different directions in order to identify the cross section of blood vessels [12], [13]. Pattern recognition techniques can be divided into supervised and unsupervised approaches. Supervised methods, such as artificial neural networks [14] or support vector machines [15], [16], exploit some prior labelling information to decide whether a pixel belongs to a vessel or not, while unsupervised algorithms [17] perform the vessel segmentation without any prior labelling knowledge. Morphological processing is based on vessels characteristics known a priori (line connected segments) and combines morphological operators to achieve the segmentation [18]-[20]. In general, techniques focused on edge detectors lack of strength in distinguishing which are desired edges and which are not, for example, in our case, 
the border of the vessels and the optic disc. Matched filters have difficulty adapting to changes in width and orientation of vessels, in all databases. Techniques that use supervised methods get a better performance than those focused on unsupervised ones and can produce very good results for healthy retinal images. However, they need a large training set of manually segmented reference images and it is difficult to automate them for any dataset. Morphological processing runs the risk of fragmenting the vessels.

As for state-of-the-art systems which compute measurements of retinal vessels, most software is focused only on calibre determination and no other measures are performed [21]-[23]. Moreover, they are not used to detect retinal vessels or measure geometrical properties automatically but the diameters of all arterioles and venules are measured by trained graders using different tools provided by the systems to make this task simpler.

The rest of the paper is organized as follows: in Section II the proposed method is presented, including material definition, theoretical background and algorithm description. Section III shows the system validation through a doubleblind study in addition to an intermediate validation of the image processing. Finally, Section IV provides discussion and Section V conclusions and some future areas for work.

\section{METHOD}

\section{A. Material}

Two different validations were performed to determine effectiveness of the proposed work. One for validating the main stage of the image processing and the other to validate the results provided by the software. For each validation, a different database was used due to its particular characteristics.

To validate the vessel segmentation, our method results were compared with a public database of hand-segmented images widely used in the literature. In the DRIVE database [24] original images and the images manually segmented by two different specialists are included. The first-observer images were taken as reference (gold standard) to calculate similarity degree between them and our segmentation.

For system validation, a set of 67 fundus images was used. These images belong to a private database of the Fundación Oftalmológica del Mediterráneo (Spain), which contains color images of 2048 x 1536 pixels. The implemented system was installed in this organization in order to be used by its clinicians in their daily practice and be able to carry out a clinic validation of the developed software based on a doubleblind study. The study was performed on 67 patients between 33 and 73 years old. Among them, a control group without previous known pathology and the study group of hypertensive patients were established. The control group was formed by 38 healthy people and the study group by 29 diseased patients previously diagnosed and treated or not for more than 5 years of evolution. Both groups were established based on the disease history of its members. Their fundus images were evaluated twice. Once, by qualified ophthalmologists who determined the presence or absence of vascular alteration through visual inspection of the original image, and another time using the developed system. A patient was considered hypertensive if the clinician detected in their fundus at least one sign of pathological arteriovenous crossing. The same tree ophthalmologists rated the entire dataset with and without the software. A minimum period of one month was established between both rates to avoid the effect of the repeated exposure to the images. The final expert rates were obtained taking the majority vote among the three clinicians.

\section{B. Theoretical background}

Mathematical morphology is a non-linear image processing methodology based on minimum and maximum operations [25] whose aim is to extract relevant structures of an image. Let $f$ be a grey scale image and $B$ be a sub-set or window, called structuring element (SE) in morphological image processing, whose shape is usually chosen according to some a priori knowledge about the geometry of the relevant and irrelevant image structures that want to be removed or kept. The two basic morphological operators are: dilation $\left(\delta_{B}(f)\right)$ and erosion $\left(\varepsilon_{B}(f)\right)$. Their purpose is to expand light or dark regions, respectively, according to the size and shape of the structuring element. Those elementary operations can be combined to obtain a new set of operators or basic filters: opening $\left(\gamma_{B}(f)\right)$ and closing $\left(\varphi_{B}(f)\right)$. Light or dark structures are respectively filtered out from the image by these operators regarding the structuring element chosen.

The method proposed in this paper applies these basic filters directly along with more complexes derived from them, such as a dual top-hat $\left(\rho_{B}(f)=\varphi_{B}(f)-f\right)$ and a supremum of openings which is defined as $\gamma_{\mathbf{B}}^{\text {sup }}(f)=\max _{i=1 \ldots N}\left\{\gamma_{B_{i}}(f)\right\}$, being $i$ the number of openings and $\mathbf{B}$ a family of linear structuring elements with different orientations. The dual tophat lets detect dark objects smaller than the SE and the supremum of openings is used when an only opening is not enough to remove certain objects because they have different orientations.

Different morphological operators that complement the previous ones are geodesic transformations. In these transformations two images are required, a marker and a reference image. The geodesic operator used in the algorithm proposed in this paper is the geodesic dilation which allows to filter objects that are present in the reference image but not in the marker. It is used to simplify the original image removing no desired objects and preserving the shape of the rest.

With the aim of detecting specific patterns in an image, other binary morphological operation can be applied, the hit-or-miss transformation (HMT) [26]. The structuring element employed in this operation is called composite structuring element since it contains two SE. The first, denoted by $B_{F G}$, defines the set of pixels that should match the foreground while the second, denoted by $B_{B G}$, defines the set of pixels that should match the background. By definition, $B_{F G}$ and $B_{B G}$ share the same origin and are disjoint sets, i.e., $B_{F G} \cap B_{B G}=0$. Depending on whether the origin belongs to $B_{F G}$ or $B_{B G}$, the HMT extracts foreground or background pixels.

The effect of these operators on an image will be shown in the next subsection where they will be applied to the images under study. 


\section{Algorithm}

The computer-aided diagnosis software presented in this paper is based on digital fundus image processing for the determination of hypertensive risk. In general, systemic diseases produce vascular changes in our blood system and usually induce specific modifications in the retinal vessels; hence, the system is focused on vessel detection and the measurement of some of their features to characterize any retinal morphological change. So, the main stages involved in the system are: firstly, vessel segmentation process; secondly, retinal tree labelling to identify the pixels that correspond to each vessel and the existing relationship between the branches; and thirdly, performing characteristic measurements on the retinal tree, using the previous information, to quantify significant changes in the vascular network. These data will be used to classify an image as belonging to a healthy patient or to other with cardiovascular risk. The mentioned stages can be observed in the block diagram of the Fig.1 where the entire image processing carried out by the proposed system is illustrated.

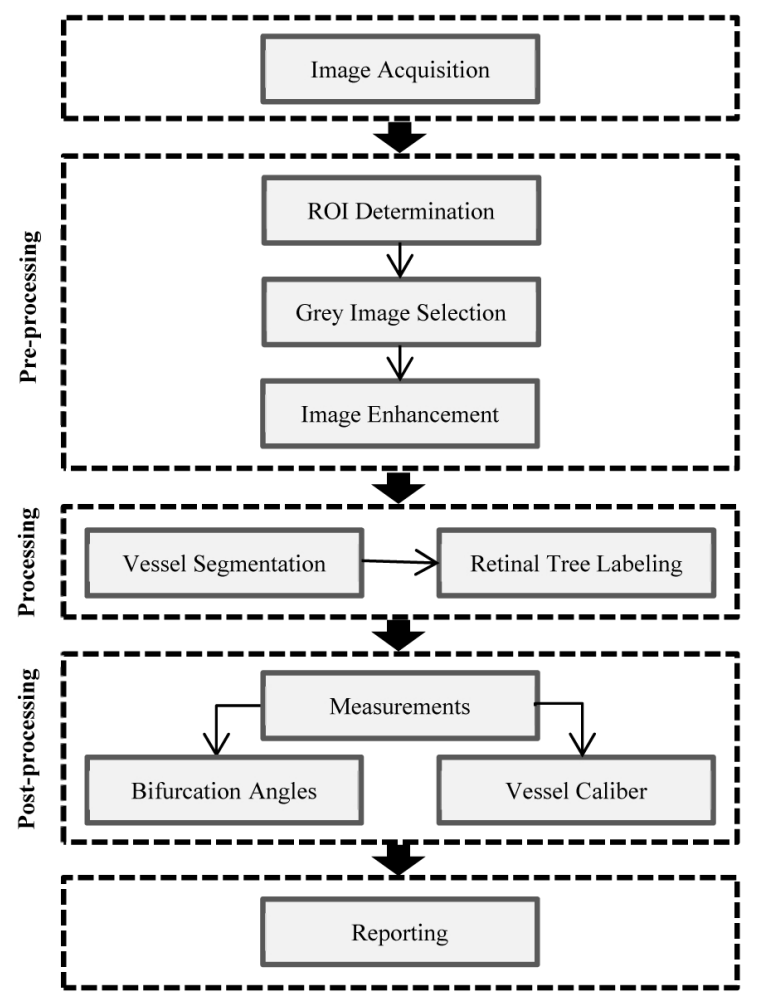

Fig. 1. Block diagram of the presented tool.

\section{1) Pre-processing}

Generally, based on a standard protocol, the measurement of retinal vessel calibres is focused on a specific region of interest (ROI) of the fundus image [21]-[23]. This area is concentric to the optic disc and it is related to with its diameter. So, for that reason, in first place, the proposed system detects the optic disc (OD) in an automatic way in order to be able to determine the ROI where all measures will be performed (Fig. 4b). The method used for the extraction of the optic disc is mainly based on mathematical morphology along with a principal component analysis (PCA) [27]. First, PCA is applied on the RGB fundus image in order to obtain a grey image in which the different structures of the retina are differentiated more clearly to get a more accurate detection of the OD. Then, the vessels are removed through inpainting technique to make the segmentation task easier. Next, a variant of the watershed transformation, the stochastic watershed transformation, followed by a stratified watershed, are implemented on a region of the original image. Finally, it must be discriminated which of the obtained watershed regions belong to the optic disc and which ones are not. A geodesic transformation and a further threshold are used to achieve that purpose.

Once the ROI has been established, although original fundus images are RGB images, the system is only drawn on monochrome images for vessel detection. They are obtained from the green band because this band provides an improved visibility of retinal blood vessels. Afterwards, an image enhancement [19] is applied to improve even more, if it is possible, their visibility.

\section{2) Processing}

The segmentation method used by the system is based on mathematical morphology [26], curvature evaluation and $\mathrm{k}$ means clustering [28] for the detection of a vascular tree [29]. First, a small opening, using a disc of radius $1\left(B_{1}\right)$ as the structuring element (SE), is performed on the enhanced green component image to fill in any gaps of the vessels which could induce errors in segmentation. In particular, the image enhancement is carried out through a local shade correction [19]. Then, a dual top-hat, with a circular SE larger than the biggest vessel $\left(B_{2}\right)$, is applied with the goal of extracting all of them and eliminating structures with high curvature that are not vessels, as occurs in the optic disc. After that, to highlight the vessels on the background, the next steps are followed. Principal curvature is calculated as the maximum eigenvalue of the Hessian matrix $(H)$ [10]. Subsequently, a reconstruction by dilation is applied to reconstruct the principal curvature from a supremum of openings which were calculated using a line of size 31 as SE every $15^{\circ}\left(\mathbf{B}_{\mathbf{3}}=\left\{B_{3_{1}}, B_{3_{2}}, \ldots, B_{3_{N}}\right\}\right.$, with $N$ the total number of openings). This operation removes any structure smaller than this SE in any orientation obtaining a cleaner background. Finally, to binarize the resulting image a $\mathrm{k}$-means clustering [28] is used with a $k$ value equals to 3 , giving rise to $\mathbf{f}_{\mathbf{k m}}=\left(f_{1}, f_{2}, f_{3}\right)$. Afterwards, a modification of the k-means output is carried out; two of the three obtained clusters are defined as vessel $\left(f_{\text {out }}=f_{1} \cup f_{2}\right)$, considering that $f_{3}$ corresponds to the background. Three classes are required because thick and thin vessels can be very different.

The next algorithm summarizes the main steps of the segmentation method and Fig. 2 depicts the resulting images.

After the vessels have been detected, they must be labelled. Retinal vascular tree labelling is focused on obtaining the skeleton of vascular tree, detecting significant points (terminal, bifurcation and crossing points) and a tracking process [29]. They are necessary steps to perform later the desired measures.

The skeleton of the vascular tree is obtained by a thinning process from the segmented binary image [26]. Next, a pruning process is applied to eliminate possible spurs and to avoid multiple paths that are inherent in a 8-connected boundary. 


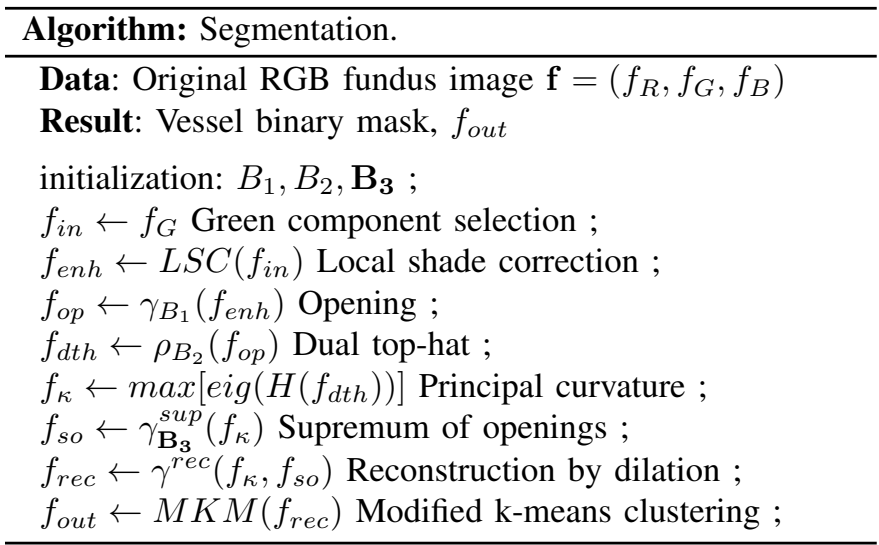

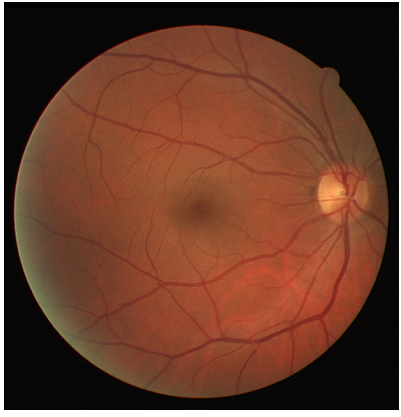

(a)

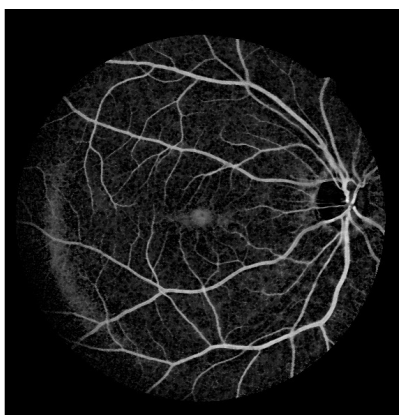

(c)

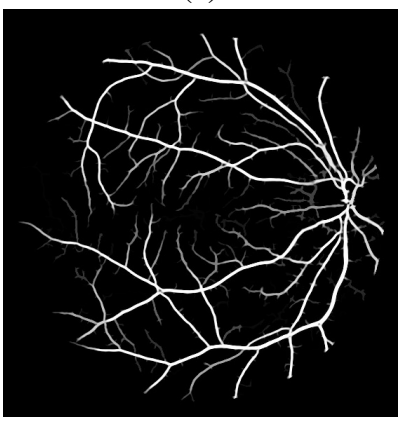

(e)

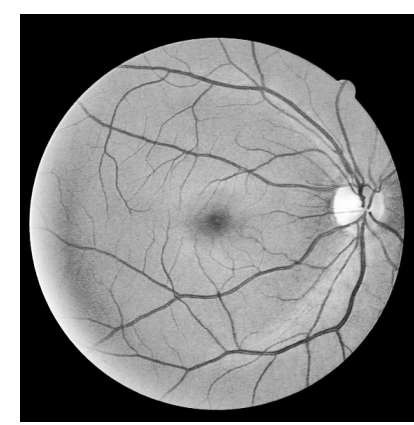

(b)

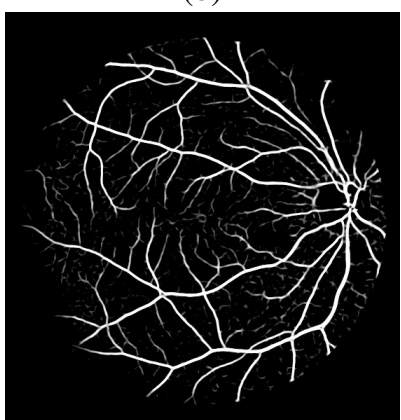

(d)

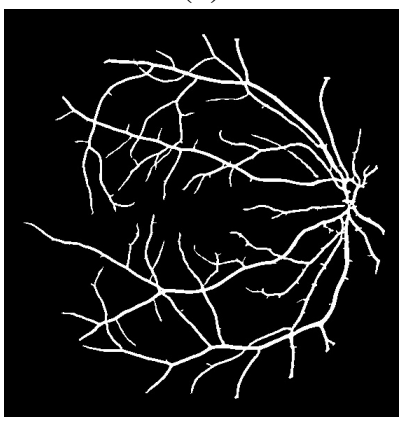

(f)
Fig. 2. Vessel segmentation steps: (a) Original fundus image, (b) Green component enhancement, (c) Dual top-hat filtering, (d) Principal curvature, (e) Reconstruction by dilation and (f) k-means clustering (segmented image).

In a vascular tree skeleton there are three types of significant points and all of them must be detected: terminal, bifurcation and crossing points. To locate the terminal and bifurcation points the hit-or-miss transform [26] is used. Fig. 3 shows the SEs used to detect these points on a skeleton. 1's define the set of pixels that should match the foreground and 0's the background. Notice that all of them must be used in all its orientations, one every $90^{\circ}$. On the other hand, to detect the crossing points it will be necessary to have a manual intervention as some of them are incorrectly defined as bifurcation points.

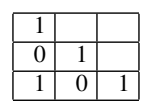

(a)

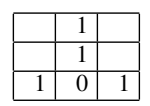

(b)

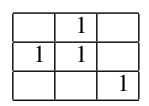

(c)

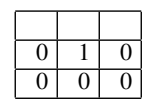

(d)
Fig. 3. Structuring elements used to detect significant points: (a-c) bifurcation points and (d) terminal points.

The tracking purpose is to analyse the direction of every pixel of the skeleton and to identify which pixels belong to each branch. The method used is proposed in [30]. The algorithm is able to specify the direction of any skeleton point from a starting point. In order to keep the relation information between the branches, a specific numbering scheme is used.

\section{3) Post-processing}

Certain geometric measurements of blood vessels can help to establish whether they have undergone morphological changes over time and facilitate disease diagnosis. The remaining parameters have been chosen due to the fact that they have particular interest for the early hypertension detection:

Bifurcation angle: Angle formed by the daughter branches for each bifurcation point. The branches are fitted for straight lines by least-squares into a circular window centred on these points.

Vessel calibre: It has been estimated as two times the average of the geodesic distance [26] calculated from the skeleton points of the branch to the edge of the corresponding vessel.

Fig. 4 shows different stages of the image processing performed by the developed software.

\section{RESULTS}

\section{A. Segmentation validation}

The performance of the vessel segmentation algorithm was evaluated based on three concepts: accuracy (Ac) and true positive (TPF) and false positive (FPF) fractions. In table I these results can be observed. In summary, the average values obtained by the proposed method are: Ac $=0.9417$, $\mathrm{TPF}=0.6570$ and $\mathrm{FPF}=0.0166$. The segmented images by the second observer was also compared with the gold standard in order to note that there are also differences between the two experts. See Table I.

In a parallel way, a study about the accuracy of different vessel segmentation methods on the same database was carried out in [31]. This study compared several approaches: matched filter [32], scale-space analysis and region growing [33], mathematical morphology and curvature estimation [18], verification-based local thresholding [11] and pixel classification [31]. Table II shows a comparison between the aforementioned methods and ours. To sum up, it can be concluded that our method has achieved a higher accuracy ratio and its 


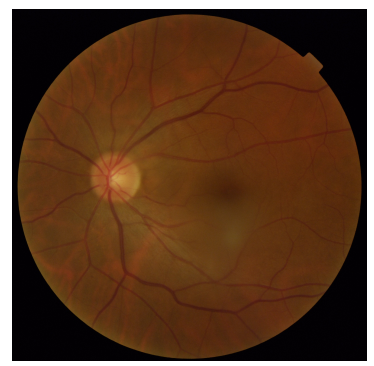

(a)

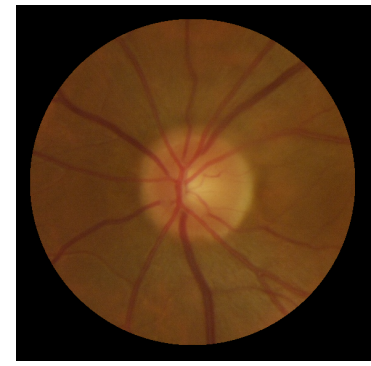

(b)

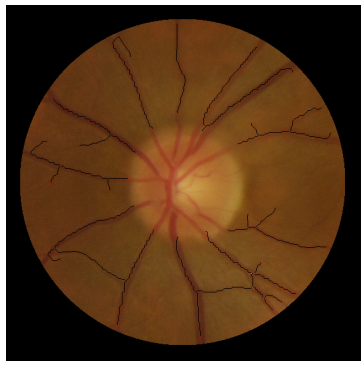

(c)

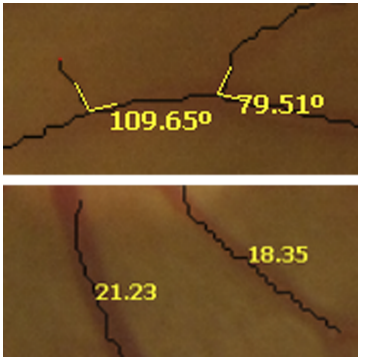

(d)

Fig. 4. Image processing performed by the developed software: (a) Original fundus image, (b) ROI determination, (c) Vessel segmentation, (d) Bifurcation angles (up) and vessel calibres (down).

false positive fraction is, even, lower than the second observer fraction. Moreover, apart from improving the accuracy ratio, it must be understood out the optic disc edge is not detected as a vessel unlike most edge detection methods, for example [10]. Figure 5 shows the resulting image of our method along with the image obtained by [10]. The non-detection of this feature is crucial to avoid multiple errors in the tracking process.

TABLE I

COMPARISON BETWEEN THE RESULTS OF THE PROPOSED METHOD AND THE $2^{\text {nd }}$ OBSERVER (AVERAGE VALUES AND STANDARD DEVIATIONS) REGARDING THE GOLD STANDARD.

\begin{tabular}{|c|c|c|}
\cline { 2 - 3 } \multicolumn{1}{c|}{} & Proposed method & $\mathbf{2}^{\text {nd }}$ observer \\
\hline Ac & $0.9417(0.0076)$ & $0.9473(0.0048)$ \\
TPF & $0.6570(0.0668)$ & $0.7757(0.0596)$ \\
FPF & $0.0166(0.0093)$ & $0.0275(0.0083)$ \\
\hline
\end{tabular}

TABLE II

ACCURACY (AVERAGE AND STANDARD DESVIATION) OF SEVERAL METHODS ON THE SAME PUBLIC DATABASE.

\begin{tabular}{|l|c|}
\cline { 2 - 2 } \multicolumn{1}{c|}{} & \multicolumn{1}{c|}{ Ac } \\
\hline Proposed method & $0.9417(0.0076)$ \\
$\mathbf{2}^{\text {nd observer }}$ & $0.9473(0.0048)$ \\
Niemeijer et al. [31] & $0.9416(0.0065)$ \\
Zana and Klein [18] & $0.9377(0.0077)$ \\
Jiang and Mojon [11] & $0.9212(0.0076)$ \\
Martinez-Perez et al. [33] & $0.9181(0.0240)$ \\
Chaudhuri et al. [32] & $0.8773(0.0232)$ \\
\hline
\end{tabular}

The optic disc segmentation is needed to establish the region of interest where the measurements are performed. In [27], a wide validation of this intermediate processing can be found.

\section{B. Computer-aided diagnosis software validation}

With the implemented tool, bifurcation angles, inside of an existing region of the original image concentric to the optic disc, were measured along with the calibre of a vein and an artery manually selected and situated at the same distance from the optic disc. Subsequently, a statistical analysis on different variables, extracted from data provided by the system, was conducted to see if any of them were able to discriminate whether a patient belonged to the group of hypertensive or without cardiovascular disease. Statistical Package for the Social Sciences (SPSS, IBM SPSS Data Collection) version 17.0 was used for this purpose.

Two parallel statistical studies were performed, one analysing the variables related to the calibre and other analysing those related to the bifurcation angles. The reason for the distinction was that only fundus images with at least five measured bifurcation angles were considered valid for this specific study. In both, the comparison between control and study groups was conducted using one-way ANOVA, where the dependent variables were each of the parameters calculated from the data provided by the software (vein calibre, artery calibre, relative calibre of the vein and artery ( $\mathrm{A} / \mathrm{V}$ ratio), average, median, deviation and variance of the branching angles, minimum and maximum angle and difference between the maximum and minimum angle) and the factor was the group formed by the control (no known disease) and study (hypertension) subgroups.

Previous to the analysis, the normality of the calculated parameters was checked using the Kolmogorov-Smirnov test. From the obtained values, it can be concluded that only the calibre of the artery $(F(1 ; 66)=4.471 ; p<0.05)$ and the ratio between the calibre of the vein and the artery $(F(1 ; 66)=$ $4.161 ; p<0.05)$ show statistically significant differences between the control and study subgroups. Afterwards, using only these parameters, the optimal threshold to separate both classes was established and then the sensitivity, specificity and accuracy of the system to discriminate between healthy patients and with hypertensive pathology were calculated.

$$
\begin{aligned}
& \text { Sensitivity }=\frac{T P}{T P+F N}=56.41 \% \\
& \text { Specificity }=\frac{T N}{T N+F P}=67.86 \%,
\end{aligned}
$$

$$
\text { Accuracy }=\frac{T P+T N}{T P+F P+F N+T N}=61.19 \%,
$$

where $T P=22, F N=17, T N=19$ and $F P=9$ are the true positives, false negatives, true negatives and false positives, respectively. If the proportion of true results of the analysed population is taken into account (i.e. the system accuracy), ophthalmologist visual diagnoses agreed with patient history $42 \%$ of the time without the computer 


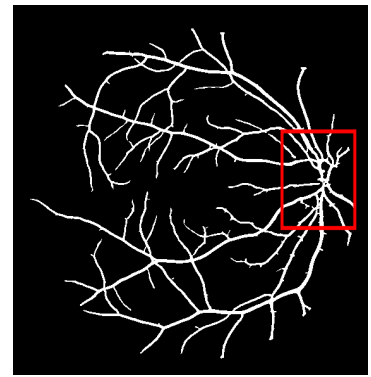

(a)

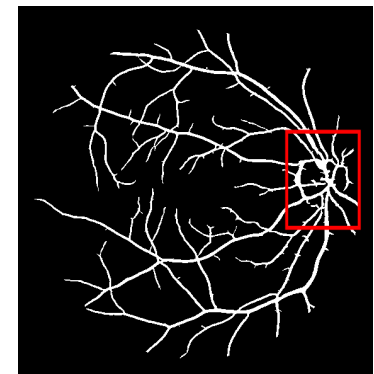

(b)

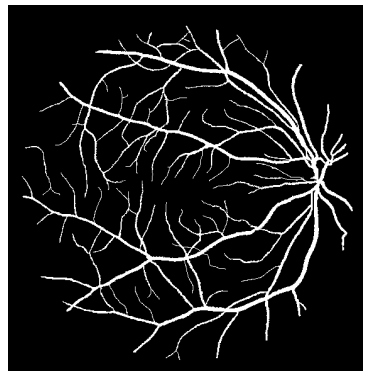

(c)

Fig. 5. Vessel segmentation comparison: (a) Proposed method, (b) [10] method and (c) Manually segmented image belonging to the DRIVE database.

assistance and that value increases up to $61 \%$ with the computer assistance. Their sensitivity and specificity are also improved from $38 \%$ and $50 \%$ to $56 \%$ and $68 \%$, respectively. Therefore, it can be concluded that the use of this computeraided diagnosis software provides an improvement of almost $20 \%$ in hypertension detection.

\section{DisCusSION}

Thanks to the software validation, it has been demonstrated that the calibre of the arteries and the relative calibre of the veins and arteries show significant differences when patients are classified either healthy or hypertensive. Despite these results, it cannot be concluded that bifurcation angles are not significant for this purpose because only $54 \%$ of the fundus of the dataset could be analysed due to the fact that the remainder contains less than 5 angles per image. Thus, the region where the measures are taken, should be enlarged or the database increased to repeat the same analysis.

Although the values of accuracy, sensitivity and specificity of the system are not too much high in the hypertension discrimination, it must be stressed that the results achieved by clinicians by visual inspection of the fundus are improved almost $20 \%$.

With regard to the obtained measures, they are accurate and reliable but also dependent on a correct skeleton detection and significant point classification.

\section{CONCLUSION}

A computer-aided diagnosis software for hypertensive risk determination has been presented. From a fundus image, the tool automatically detects blood vessels and allows measurement of bifurcation angles and selection of branches to determine their calibre. These data facilitate expert medical diagnosis and study of the progression of the disease. In particular, the software has been used by expert ophthalmologists to help them to discriminate between a normal vascularization and cardiovascular pathology. It has improved by almost $20 \%$ the accuracy, sensitivity and specificity in hypertension detection achieved by direct visual inspection of the fundus.

In future work, the system will be applied to analyse the retinal microvascular architecture of children with low birthweight in order to establish the relationship between the measurements obtained in these children and diseases such as hypertension and cardiovascular problems in adult life, using it as a prognostic marker of cardiovascular risk. The base of this study (carried out by the Department of Paediatrics of General Hospital of Valencia (Spain)) is that bifurcation angles, which are determined at birth, are predictors of future development of hypertension and cardiovascular disease. Moreover, the analysis of the angles should be repeated enlarging the region of measures or increasing the database, as has been mentioned in the previous section. Regarding the values of sensitivity and specificity of the system, they could be improved if other classifiers were used, such as SVM, among others, or by increasing the database size. In addition, the study to correlate segmentation parameters and hypertensive pathologies will be widen.

\section{ACKNOWLEDGMENT}

This work has been funded by the project IMIDTA/2010/47 and partially by projects Consolider-C (SEJ2006 14301/PSIC),CIBER of Physiopathology of Obesity and Nutrition, an initiative of ISCIII and Excellence Research Program PROMETEO (Generalitat Valenciana. Conselleria de Educación, 2008-157). We would like to express our deep gratitude to Imex Clinic S.L., the Department of Paediatrics of General Hospital of Valencia and the Fundación Oftalmológica del Mediterráneo for its participation in the project.

\section{REFERENCES}

[1] H. M. Herbert, K. Jordan, and D. W. Flanagan, "Is screening with digital imaging using one retinal view adequate?" Eye, no. 4, p. 497500, 2003.

[2] L. Verma, G. Prakash, and H. K. Tewari, "Diabetic retinopathy: time for action. No complacency please!" Bulletin of the World Health Organization, vol. 80, pp. 419 - 419, 2002.

[3] K. Goatman, A. Charnley, L. Webster, and S. Nussey, "Assessment of automated disease detection in diabetic retinopathy screening using twofield photography," PLoS ONE, vol. 6, no. 12, e27524, 2011.

[4] M. D. Abràmoff, J. M. Reinhardt, S. R. Russell, J. C. Folk, V. B. Mahajan, M. Niemeijer, and G. Quellec, "Automated early detection of diabetic retinopathy," Ophthalmology, vol. 117, no. 6, pp. 1147 1154, 2010.

[5] T. T. Nguyen, J. J. Wang, and T. Y. Wong, "Retinal vascular changes in pre-diabetes and prehypertension: New findings and their research and clinical implications," Diabetes Care, vol. 30, no. 10, pp. 2708-2715, 2007.

[6] S. L. Rogers, G. Tikellis, N. Cheung, R. Tapp, J. Shaw, P. Z. Zimmet, P. Mitchell, J. J. Wang, and T. Y. Wong, "Retinal arteriolar caliber predicts incident retinopathy: the australian diabetes, obesity and lifestyle (ausdiab) study." Diabetes Care, vol. 31, no. 4, pp. 761-3, 2008.

[7] N. Cheung and T. Y. Wong, "Diabetic retinopathy and systemic vascular complications," Progress in Retinal and Eye Research, vol. 27, no. 2, pp. $161-176,2008$. 
[8] M. D. Abràmoff, M. K. Garvin, and M. Sonka, "Retinal imaging and image analysis," Biomedical Engineering, IEEE Reviews in, vol. 3, pp. 169-208, Dec. 2010.

[9] M. M. Fraz, P. Remagnino, A. Hoppe, B. Uyyanonvara, A. R. Rudnicka, C. G. Owen, and S. A. Barman, "Blood vessel segmentation methodologies in retinal images-a survey," Computer methods and programs in biomedicine, vol. 108, no. 1, pp. 407-433, 2012.

[10] M. E. Martinez-Perez, A. D. Hughes, S. A. Thom, A. A. Bharath, and K. H. Parker, "Segmentation of blood vessels from red-free and fluorescein retinal images," Medical Image Analysis, vol. 11, pp. 47-61, 2007.

[11] X. Jiang and D. Mojon, "Adaptive local thresholding by verificationbased multithreshold probing with application to vessel detection in retinal images," IEEE Trans. Pattern Anal. Mach. Intell., vol. 25, pp. 131-137, January 2003.

[12] A. Hoover, V. Kouznetsova, and M. Goldbaum, "Locating blood vessels in retinal images by piecewise threshold probing of a matched filter response," IEEE Transactions on Medical Imaging, vol. 19, pp. 203$210,2000$.

[13] M. Al-Rawi, M. Qutaishat, and M. Arrar, "An improved matched filter for blood vessel detection of digital retinal images," Computers in Biology and Medicine, vol. 37, no. 2, pp. 262-267, 2007.

[14] C. Sinthanayothin, J. F. Boyce, H. L. Cook, and T. H. Williamson, "Automated localisation of the optic disc, fovea, and retinal blood vessels from digital colour fundus images," British Journal of Ophthalmology, vol. 83, pp. 902-910, 1999.

[15] E. Ricci and R. Perfetti, "Retinal blood vessel segmentation using line operators and support vector classification," Medical Imaging, IEEE Transactions on, vol. 26, no. 10, pp. 1357-1365, 2007.

[16] D. Marín, A. Aquino, M. E. Gegúndez-Arias, and J. M. Bravo, "A new supervised method for blood vessel segmentation in retinal images by using gray-level and moment invariants-based features," Medical Imaging, IEEE Transactions on, vol. 30, no. 1, pp. 146-158, 2011.

[17] G. B. Kande, P. V. Subbaiah, and T. S. Savithri, "Unsupervised fuzzy based vessel segmentation in pathological digital fundus images," Journal of medical systems, vol. 34, no. 5, pp. 849-858, 2010.

[18] F. Zana and J. C. Klein, "Segmentation of vessel-like patterns using mathematical morphology and curvature evaluation," IEEE Transactions on Image Processing, vol. 10, pp. 1010-1019, 2001.

[19] T. Walter and J. C. Klein, "A computational approach to diagnosis of diabetic retinopathy," in Proceedings of the 6th Conference on Systemics Cybernetics and Informatics (SCI), 2002, pp. 521-526.

[20] K. Sun, Z. Chen, S. Jiang, and Y. Wang, "Morphological multiscale enhancement, fuzzy filter and watershed for vascular tree extraction in angiogram," Journal of medical systems, vol. 35, no. 5, pp. 811-824, 2011.

[21] T. Y. Wong, M. D. Knudtson, R. Klein, B. E. K. Klein, S. M. Meuer, and L. D. Hubbard, "Computer-assisted measurement of retinal vessel diameters in the beaver dam eye study: methodology, correlation between eyes, and effect of refractive errors." Ophthalmology, vol. 111, no. 6, pp. 1183-1190, 2004.

[22] M. D. Knudtson, K. E. Lee, L. D. Hubbard, T. Y. Y. Wong, R. Klein, and B. E. Klein, "Revised formulas for summarizing retinal vessel diameters." Current Eye Research, vol. 27, no. 3, pp. 143-149, Sep. 2003.

[23] L. D. Hubbard, R. J. Brothers, W. N. King, L. X. Clegg, R. Klein, L. S. Cooper, A. Sharrett, M. D. Davis, and J. Cai, "Methods for evaluation of retinal microvascular abnormalities associated with hypertension/sclerosis in the atherosclerosis risk in communities study," Ophthalmology, vol. 106, no. 12, pp. 2269 - 2280, 1999.

[24] J. Staal, M. Abramoff, M. Niemeijer, M. Viergever, and B. van Ginneken, "Ridge-based vessel segmentation in color images of the retina," Medical Imaging, IEEE Transactions on, vol. 23, no. 4, pp. 501 -509, april 2004.

[25] J. Serra, Image Analysis and Mathematical Morphology. London: Ac. Press, 1982, vol. I.

[26] P. Soille, Morphological Image Analysis: Principles and Applications, 2nd ed. Springer-Verlag New York, Inc., 2003.

[27] S. Morales, V. Naranjo, J. Angulo, and M. Alcaniz, "Automatic detection of optic disc based on pca and mathematical morphology," Medical Imaging, IEEE Transactions on, vol. 32, no. 4, pp. 786-796, 2013.

[28] J. B. MacQueen, "Some methods for classification and analysis of multivariate observations," in Proc. of the fifth Berkeley Symposium on Mathematical Statistics and Probability, vol. 1, 1967, pp. 281-297.

[29] S. Morales, V. Naranjo, J. Angulo, J. J. Fuertes, and M. A. niz, "Segmentation and analysis of retinal vascular tree from fundus images processing," in International Conference on Bio-inspired Systems and Signal Processing (BIOSIGNALS 2012). SciTePress, February 2012, pp. $321-324$.

[30] M. E. Martinez-Perez, A. D. Hughes, A. V. Stanton, S. A. Thorn, N. Chapman, A. A. Bharath, and K. H. Parker, "Retinal vascular tree morphology: A semi-automatic quantification," in Biomedical Engineering, vol. 49, no. 8, 2002.

[31] M. Niemeijer, J. Staal, B. van Ginneken, M. Loog, and M. Abramoff, "Comparative study of retinal vessel segmentation methods on a new publicly available database," in SPIE Medical Imaging, vol. 5370, 2004, pp. 648-656.

[32] S. Chaudhuri, S. Chatterjee, N. Katz, M. Nelson, and M. Goldbaum, "Automatic detection of the optic nerve in retinal images," in IEEE International Conference on Image Processing, vol. 1, 1989, pp. 1-5.

[33] M. Martinez-Perez, A. Hughes, A. Stanton, S. Thom, A. Bharath, and K. Parker, "Retinal blood vessel segmentation by means of scalespace analysis and region growing," in Medical Image Computing and Computer-Assisted Intervention MICCAI 99, ser. Lecture Notes in Computer Science, 1999, vol. 1679, pp. 90-97. 